\title{
Disordered Fermi Liquid in Epitaxial Graphene from Quantum Transport Measurements
}

\author{
Samuel Lara-Avila, ${ }^{1, *}$ Alexander Tzalenchuk, ${ }^{2}$ Sergey Kubatkin, ${ }^{1}$ Rositza Yakimova, ${ }^{3}$ T. J. B. M. Janssen, ${ }^{2}$ \\ Karin Cedergren, ${ }^{1}$ Tobias Bergsten, ${ }^{4}$ and Vladimir Fal'ko ${ }^{5}$ \\ ${ }^{1}$ Department of Microtechnology and Nanoscience, Chalmers University of Technology, Göteborg, S-41296, Sweden \\ ${ }^{2}$ National Physical Laboratory, Teddington, TW110LW, United Kingdom \\ ${ }^{3}$ Department of Physics, Chemistry and Biology (IFM), Linköping University, Linköping, S-58183, Sweden \\ ${ }^{4}$ SP Technical Research Institute of Sweden, S-50115, Sweden \\ ${ }^{5}$ Physics Department, Lancaster University, Lancaster, LA14YB, United Kingdom
}

(Received 10 June 2011; published 12 October 2011)

\begin{abstract}
We have performed magnetotransport measurements on monolayer epitaxial graphene and analyzed them in the framework of the disordered Fermi liquid theory. We have separated the electron-electron and weak-localization contributions to resistivity and demonstrated the phase coherence over a micrometer length scale, setting the limit of at least $50 \mathrm{ps}$ on the spin relaxation time in this material.
\end{abstract}

DOI: 10.1103/PhysRevLett.107.166602

PACS numbers: 72.80.Vp, 71.70.Ej, 73.20.Fz, 73.43.Qt

Epitaxial graphene synthesized on the Si-terminated face of $\mathrm{SiC}(\mathrm{SiC} / \mathrm{G})$ has uniquely demonstrated the ability to supersede conventional semiconductors as the system of choice for fast analogue transistors [1], quantum metrology [2-5], and is emerging as a suitable platform for such disruptive technologies as spintronics. In $\mathrm{SiC} / \mathrm{G}$, the twodimensional system is formed by a conducting graphene layer situated on top of a nonconducting buffer carbon layer, covalently bonded to $\mathrm{SiC}$. The interplay between these two layers, unavailable in graphene flakes, graphene on the $\mathrm{C}$ face of $\mathrm{SiC}$ or conventional semiconductor-based 2D gases, makes $\mathrm{SiC} / \mathrm{G}$ a system full of rich new physics. Therefore a detailed understanding of electron scattering and localization in $\mathrm{SiC} / \mathrm{G}$ is important; however, no detailed analysis of charge and spin transport in this promising material have been reported until now.

Specifically for graphene, the type of scattering can be revealed by a detailed analysis of the weak-localization effect (WL) [6,7], due to the deep relation between the relativistic character of charge carriers in the two valleys of graphene band structure and its sublattice symmetry $[8,9]$. Generally, WL is manifested by a magnetic-fielddependent correction to the conductivity at low temperatures resulting from the constructive interference of electrons circling (clockwise and anticlockwise) in a diffusive random walk trajectory [10]. We have separated the contribution to electron scattering due to coupling of graphene to the buffer carbon layer leading to the local violation of the symmetry of graphene lattice and intervalley scattering. Besides WL, the conductivity in disordered conductors is also affected by the interference effect between the electron scattering from defects and Friedel oscillations of the electron density, created upon screening of the defects perturbations by electrons in the Fermi sea: the Altshuler-Aronov (AA) interaction correction to conductivity [11]. Based on the analysis of these two quantum transport effects observed in this work, we find that in
$\mathrm{SiC} / \mathrm{G}$ the intervalley scattering rate $\tau_{i}^{-1}$ is much slower than the momentum relaxation rate $\tau_{\text {tr }}^{-1}$, while scattering from the sublattice-symmetry-breaking perturbations $\tau_{*}^{-1}$, is comparable to $\tau_{\text {tr }}^{-1}$ [Fig. 1(a)]. This points towards $\mathrm{SiC} / \mathrm{G}$ conductivity being limited by charge disorder created, presumably, by donors in the buffer layer. After unwinding the AA correction from the WL contribution to the conductivity, we confirm that electrons in epitaxial
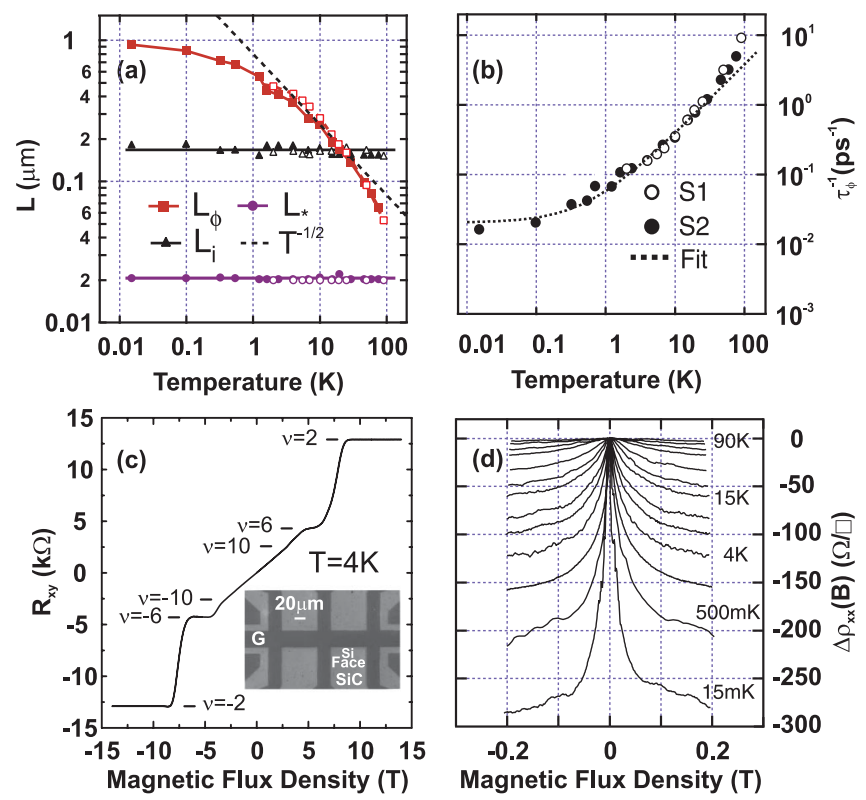

FIG. 1 (color). Magnetotransport measurements in monolayer $\mathrm{SiC} / \mathrm{G}$. (a) Temperature dependence of the characteristic scattering lengths and (b) decoherence rate $\left(\tau_{\phi}^{-1}\right)$ for samples $S 1$ (open markers) and $S 2$ (solid markers). Dotted line is the fit to $\tau_{\phi}^{-1}=\tau_{s}^{-1}+A T$, with $\tau_{s}^{-1}=0.02 \mathrm{ps}^{-1}$ and $A=0.036 \mathrm{ps}^{-1} \mathrm{~K}^{-1}$. (c) Half-integer QHE proving the monolayer nature of $\mathrm{SiC} / \mathrm{G}$. Inset: Micrograph of one of the devices. (d) Weak localization peak at different temperatures. 
graphene demonstrate all the known attributes of a disordered Fermi liquid. We note that information about the nature of disorder in graphene can in principle be obtained from the analysis of the density dependence of the Drude resistivity and quantum corrections to it. In graphene, the dominance of scattering from Coulomb impurities (e.g., donors in or on $\mathrm{SiC}$ ) is, usually, signaled by the linear dependence of conductivity on the carrier density [12-14], but in epitaxial graphene it is extremely difficult to change the carrier density broadly enough to perform such a test [15].

More intricate details of scattering of electrons can be extracted from the analysis of the temperature-dependent curvature of magnetoresistance (MR) at $B=0$, which quantifies the decoherence rate in the structure. In particular, the elastic spin relaxation caused by spin-orbit coupling or scattering of electrons manifests itself in the change of the sign of the low-temperature MR near $B=0$, from negative (specific for the WL effect) to positive, characteristic of weak antilocalization (WAL) [16]. Also, the spin relaxation due to the electron spin-flip scattering from localized magnetic moment of spin-carrying defects results in some additional decoherence leading to a dependence $\tau_{\phi}^{-1}=\tau_{s}^{-1}+A T$ of the decoherence rate upon decreasing temperature. To mention, the MR data reported in this work do not show any sign of WAL behavior (unlike graphene on $\mathrm{C}$ face of $\mathrm{SiC}[17,18]$ ); however, the measured decoherence time is at least $\tau_{s} \sim 50 \mathrm{ps}$ at the lowest temperatures [Fig. 1(b)]. On the basis of this, here we claim the first indirect measurement of spin relaxation in epitaxial graphene induced by magnetic defects (though we are not able, yet, to pinpoint the origin of such defects) and set the limit of at least $50 \mathrm{ps}$ on any possible spin-orbit-induced spin relaxation time.

We report magnetotrasport measurements on two large area $(l=160 \mu \mathrm{m}, w=35 \mu \mathrm{m})$ Hall bars ( $S 1$ and $S 2)$ patterned using standard $e$-beam lithography on different wafers of monolayer $\mathrm{SiC} / \mathrm{G}$, encapsulated with a polymer bilayer to improve temporal stability and doping homogeneity, and exposed to Deep-UV to tune the carrier concentration [3]. Low-temperature Hall mobilities and carrier concentrations are $\mu=7300 \mathrm{~cm}^{2} \mathrm{~V}^{-1} \mathrm{~s}^{-1}$, $n=4 \times 10^{11} \mathrm{~cm}^{-2}$ and $\mu=5800 \mathrm{~cm}^{2} \mathrm{~V}^{-1} \mathrm{~s}^{-1}, \quad n=$ $6 \times 10^{11} \mathrm{~cm}^{-2}$ for samples $S 1$ and $S 2$, respectively. Thanks to a large sample size [see Fig. 1(c)], our measurements are not obscured by mesoscopic effects and could be extended down to electron temperatures as low as $25 \mathrm{mK}$ in a well-filtered cryostat [19].

In the quantum limit the measured longitudinal (sheet) resistance $\rho_{x x}$ is expected to have the form

$$
\rho_{x x}=\tilde{\rho}_{x x}+\Delta \rho_{\mathrm{WL}}+\Delta \rho_{e-e}
$$

where $\tilde{\rho}_{x x}$ is the Drude (sheet) resistance, $\Delta \rho_{\mathrm{WL}}$ is the correction due to localization effects and $\Delta \rho_{e-e}$ is the correction due to electron-electron interactions. We decouple these quantum-mechanical corrections by subtracting analytically and eliminating experimentally the WL contributions to $\rho_{x x}$. The full WL correction $\Delta \rho_{\mathrm{WL}-\mathrm{Fit}}$ is found by fitting MR to the weak-localization theory of graphene [8], while the experimental suppression of WL is achieved by applying a transversal magnetic field up to $\bar{B}=1 \mathrm{~T}$ (where bar denotes a constant quantity) such that $\Delta \rho_{\mathrm{WL}-\mathrm{Exp}}=0$. If the WL correction is subtracted properly, the quantity $\tilde{\rho}_{x x}=\rho_{x x}-\Delta \rho_{\mathrm{WL}}$, where $\Delta \rho_{\mathrm{WL}}$ is either analytical or experimental, would contain only the $e$-e corrections, which can be compared with predictions of the AA theory.

The zero field, temperature-dependent analytic correction due to localization effects in graphene reads [8]

$$
\begin{aligned}
\Delta \rho_{\mathrm{WL}-\mathrm{Fit}}(B=0, \bar{T})= & \frac{e^{2} \rho^{2}}{\pi h}\left[\ln \left(1+2 \frac{\tau_{\phi}}{\tau_{i}}\right)\right. \\
& \left.-2 \ln \left(\frac{\tau_{\phi} / \tau_{\mathrm{tr}}}{1+\tau_{i} / \tau_{*}}\right)\right],
\end{aligned}
$$

where the relaxation time is calculated as $\tau_{\text {tr }}=l / v_{F}=$ $h /\left(2 e^{2} \rho_{x x} v_{F} \sqrt{\pi n}\right) \approx 0.05$ ps and the scattering times $\tau_{\phi}$, $\tau_{i}, \tau_{*}$ are found by fitting the measured $\rho_{x x}(B, \bar{T})$ to (3)

$$
\begin{aligned}
\frac{\Delta \rho(B, \bar{T})}{\rho^{2}}= & -\frac{e^{2}}{\pi h}\left[F\left(\frac{B}{B_{\phi}}\right)-F\left(\frac{B}{B_{\phi}+2 B_{i}}\right)\right. \\
& \left.-2 F\left(\frac{B}{B_{\phi}+B_{*}}\right)\right]
\end{aligned}
$$

with $F(z)=\ln (z)+\Psi\left(\frac{1}{2}+\frac{1}{z}\right), \tau_{\phi, i, *}=\frac{\hbar}{4 \mathrm{De}} B_{\phi, i, *}^{-1}$ and $D=$ $v_{F} l / 2$. From the measurements it was found that WL effects are present in all the temperature range being naturally stronger at lower temperatures [Fig. 1(b)]. Fits were performed in the range $B \leq|200| \mathrm{mT}$, field at which the transport field $\left(B_{\mathrm{tr}}=\hbar / 2 e l^{2}\right)$ becomes comparable to the mean free path in our samples $(l \approx 55 \mathrm{~nm}$ for $S 1$ and $l \approx 44 \mathrm{~nm}$ for $S 2$ ).

The values of the characteristic scattering lengths $L_{\phi, i, *}=\sqrt{D \tau_{\phi, i, *}}$, resulting from the fit to the WL theory are presented in Fig. 1(a); the fastest scattering process determining the electron momentum relaxation rate is scattering on donors on the $\mathrm{SiC}$ surface, whereas intervalley scattering is much slower. The intervalley scattering is quantified in terms of the relation between the intervalley length $L_{i}$, and the electron mean free path $l: L_{i} / l=$ $\sqrt{\left(\tau_{i} / 2 \tau_{\text {tr }}\right)}$. The observed large values of $L_{i} / l \approx 3$ for $S 1$ and $L_{i} / l \approx 6$ for $S 2$ confirm that the graphene monolayer is essentially decoupled from the underlying $\mathrm{SiC}$ lattice. In the same figure we show the temperature dependence of the coherence length $L_{\phi}$. At $T=15 \mathrm{mK}, L_{\phi}$ reaches the micrometer range for $S 2$, similar to observations in graphene flakes [6]. Measuring $S 2$ in a well-filtered dilution fridge using current levels down to $50 \mathrm{pA}$ to avoid excessive Joule heating we did not find any indication of the crossover to antilocalization nor saturation of $L_{\phi}$, 
although a slower temperature dependence was observed for $T<2 \mathrm{~K}$. This is in contrast to the saturation of $L_{\phi}$ reported in Refs. $[6,18,20]$. $S 1$ was measured in an unfiltered fridge down to $1 \mathrm{~K}$.

The mechanism of decoherence can be understood through the temperature and magnetic field dependence of the full resistivity tensor for $\mathrm{SiC} / \mathrm{G}$, presented in Fig. 2. The temperature dependence of the WL-corrected longitudinal resistivity shown as $\Delta \tilde{\rho}_{x x}=\tilde{\rho}_{x x}-\tilde{\rho}_{x x}(T=2 \mathrm{~K})$ and the corresponding longitudinal conductivity are shown in Fig. 2(a). The plot labeled "WL-corrected" was obtained by subtracting analytically the WL correction, (2), and it is observed that WL is effectively suppressed at $B=1 \mathrm{~T}$, since $\Delta \tilde{\rho}_{x x-\mathrm{Fit}} \approx \Delta \tilde{\rho}_{x x-\text { Exp }}$. Higher magnetic fields were avoided due to the onset of Shubnikovde Haas oscillations, which complicate the analysis. The transversal components are presented in Fig. 2(b), for which we observed a logarithmic increase of the Hall coefficient at low temperatures, independently of magnetic field [Fig. 2(b), top]. The product $\omega_{c} \tau_{\text {tr }}$, with $\omega_{c}=$ $e B v_{F} / \hbar \pi \sqrt{n}$, is independent of temperature but increases for higher magnetic fields, as shown in terms of $\sigma_{x y}(\bar{B}, T)$ in Fig. 2(b), bottom. The increase (decrease) of both longitudinal and transversal resistivities (conductivities) $\propto T$ at high temperatures $(T>40 \mathrm{~K})$ can be explained in terms of phonon scattering [21], as the Bloch-Grüneisen
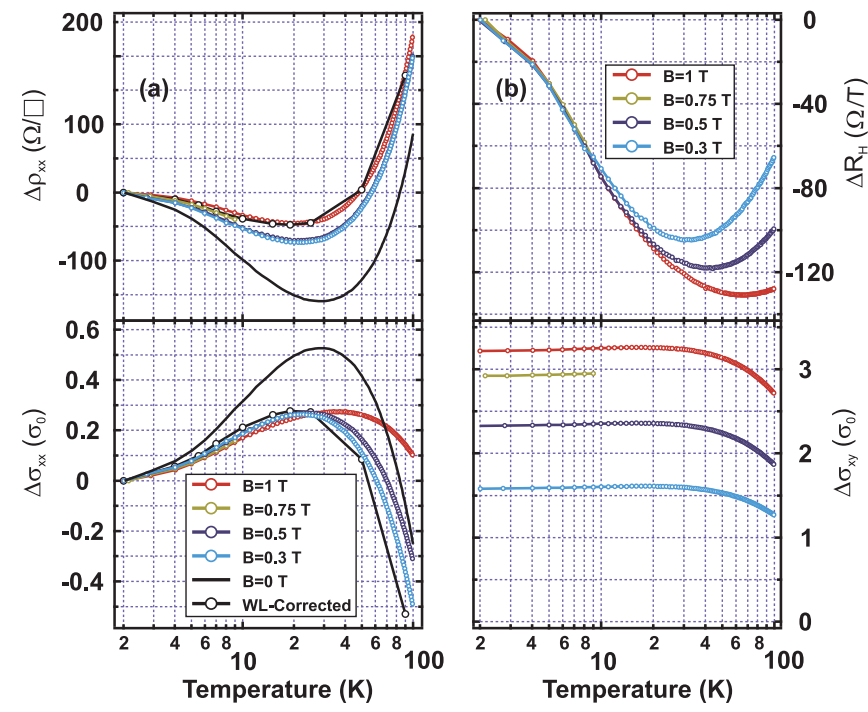

FIG. 2 (color). Magnetic field and temperature dependence of the full resistivity tensor for sample $S 1$. (a), top. Temperature dependence of $\tilde{\rho}_{x x}$ where WL effects have been suppressed analytically (labeled as "WL corrected") and experimentally. WL was substantially suppressed at $B=300 \mathrm{mT}$ and for $B=1 \mathrm{~T}, \Delta \tilde{\rho}_{\mathrm{WL}-\mathrm{Fit}} \approx \Delta \tilde{\rho}_{\mathrm{WL}-\mathrm{Exp}}$. (a), bottom. The corresponding conductivity, $\sigma_{x x}=\tilde{\rho}_{x x} /\left(\tilde{\rho}_{x x}^{2}+\rho_{x y}^{2}\right)$, in units of $\sigma_{0}=$ $2 e^{2} h \approx(12906.4 \Omega)^{-1}$. (b), top. Temperature dependence of the Hall coefficient $R_{H}$ and the transversal conductivity (bottom) $\sigma_{x y}=\rho_{x y} /\left(\tilde{\rho}_{x x}^{2}+\rho_{x y}^{2}\right)$ in units of $\sigma_{0}$. temperature for our sample, $T<T_{\mathrm{BG}}=2 v_{\mathrm{ph}} E_{F} /\left(\hbar k_{B} v_{F}\right) \approx$ $34 \mathrm{~K}$, with $V_{\mathrm{ph}}=2 \times 10^{4} \mathrm{~ms}^{-1}$ the phonon velocity, $E_{F}$ the Fermi level of graphene, and $v_{F}=10^{6} \mathrm{~ms}^{-1}$ the Fermi velocity.

For the low-temperature limit, $T<T_{\mathrm{BG}}$, where the phase coherence length decays as $\propto 1 / \sqrt{T}, \tau_{\phi}^{-1} \propto T$ and $R_{H}$ changes with temperature while $\sigma_{x y}$ is constant we found that our measurements for the two samples can be described by the AA theory for disordered systems [11]. According to this, in the presence of electron-electron interactions, corrections to the Hall coefficient will emerge

$$
\frac{\Delta R_{H}}{R_{H}}=\gamma \frac{\Delta R_{x x}}{R_{x x}},
$$

where $\gamma$ is sensitive to the details of electron scattering in the system: $\gamma=0$ in the absence of electron interactions [22], $\gamma=2$ in the presence of electron interactions, and $\gamma>2$ for the cases of electron interactions with spin-orbit coupling. Therefore, $\gamma$ becomes an additional tool to study corrections to $\rho_{x x}$, and we use it in combination with experimental data of $\Delta \tilde{\rho}_{x x}(\bar{B}, T)$ and $\Delta \tilde{\sigma}_{x x}(\bar{B}, T)$ to unequivocally distinguish electron interactions as the main source of decoherence. The temperature and magnetic field dependence of $\gamma$, extracted for both the analytic and experimental suppression of WL effects, are presented for the two samples in Fig. 3, top. For sample $S 2, \gamma \approx 2$ and for sample $S 1$ it exceeds the value of 3, probably due to the fact that at $B=1 \mathrm{~T}, \omega_{c} \tau_{\text {tr }} \approx 0.88$, close to the limit of the diffusive treatment of the problem.

The strength of the electron-electron interactions is quantified following the analysis presented in Refs. [11,23]. The temperature dependence of the WLcorrected conductivity for the two samples, $\Delta \tilde{\sigma}_{x x}=$ $\delta \sigma_{x x}(T)-\delta \sigma_{x x}(T=2 \mathrm{~K})$ (Fig. 3, bottom), was found to follow a logarithmic dependence with temperature according to

$$
\Delta \sigma(T)=-A\left(F_{0}^{\sigma}\right) \frac{e^{2}}{2 \pi^{2} \hbar} \ln \left(\frac{\hbar}{k_{B} T \tau_{\mathrm{tr}}}\right),
$$

where $A\left(F_{0}^{\sigma}\right)$ reflects the strength of the interaction, and $F_{0}^{\sigma}$ is the Fermi liquid constant. All WL-corrected measurements for each sample are fitted to the same slope $\left[A\left(F_{0}^{\sigma}\right) \approx 0.758\right.$ for $S 1$ and $A\left(F_{0}^{\sigma}\right) \approx 0.686$ for $\left.S 2\right]$, from where the Fermi liquid constant is found using [11,23]

$$
A\left(F_{0}^{\sigma}\right)=1+c\left(1-\frac{\ln \left(1+F_{0}^{\sigma}\right)}{F_{0}^{\sigma}}\right)
$$

In this case, the first term is a singlet channel and $c$ represents the contribution of $c$ triplet channels, which in graphene is $c=15$ due to fourfold spin degeneracy of two interacting electrons and an additional fourfold degeneracy due to two valleys. It has been pointed out that for graphene in the regime $\frac{\hbar}{k_{B} \tau_{i}}<T<\frac{\hbar}{k_{B} \tau_{*}}(8 \mathrm{~K}<T<600 \mathrm{~K})$ two electrons from different valleys give no contribution and as a 


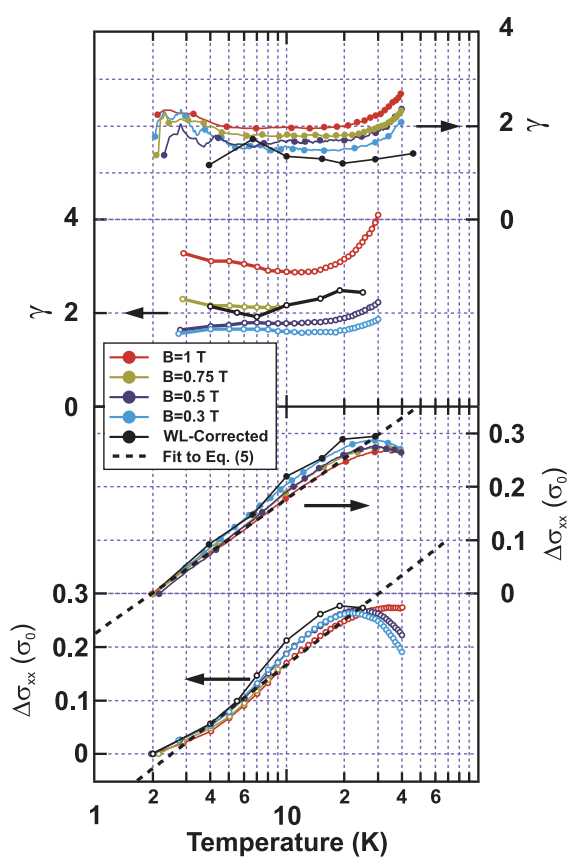

FIG. 3 (color). The conductivity in $\mathrm{SiC} / \mathrm{G}$ as described by the AA theory for electron transport in disordered systems. Top: The finite ratio $\gamma=\left(\Delta \tilde{R}_{H} / R_{H}\right) /\left(\Delta \tilde{\rho}_{x x} / \tilde{\rho}_{x x}\right)$ for the analytical and experimental WL corrected $\rho_{x x}$ confirms unequivocally the presence of electron-electron interactions in $\mathrm{SiC} / \mathrm{G}$ for the range $2 \mathrm{~K}<T<30 \mathrm{~K}$ for the two samples, $S 1$ (open circles) and $S 2$ (solid circles). Bottom: The WL-corrected conductivity displays a logarithmic dependence in temperature out of which the strength of the electron interaction can be extracted. For $S 1$, $A\left(F_{0}\right) \approx 0.758$ yielding $F_{0} \approx-0.066$ while for $S 2 F_{0} \approx-0.085$ for $A\left(F_{0}\right) \approx 0.686$ using $c=7$ triplet channels, implying the high- $\kappa$ substrate $\left(\kappa_{\mathrm{SiC}} \approx 10\right)$ does not screen interactions efficiently.

consequence $c=7$. The value for $F_{0}$ is found to be in the range $F_{0} \approx-0.066$ for $S 1$ and $F_{0} \approx-0.085$ for $S 2$. This value is comparable to $-0.13<F_{0}<-0.08$ obtained previously for flakes [23] and its low magnitude has been explained as a consequence of chiral carriers, which reduces the angle for electron-electron scattering.

In summary, we presented a comprehensive analysis of two quantum transport phenomena in epitaxial graphene grown on the Si-terminated face of $\mathrm{SiC}$-the AronovAltshuler $e-e$ interaction correction and WL contribution to resistivity. The analysis of the AA correction has confirmed that electrons in epitaxial graphene display all the attributes of a disordered Fermi liquid. The analysis of WL has enabled us to determine that the symmetry-breaking disorder and intervalley scattering play a lesser role in determining epitaxial graphene resistivity than scattering from donors on $\mathrm{SiC}$ surface. Moreover, the analysis of the temperature dependence of the decoherence rate extracted from the WL MR has enabled us to establish spin relaxation of electrons, at the time scale of $\sim 50 \mathrm{ps}$, which we attribute to the presence of local magnetic moments of defects in or under graphene. The latter conclusion is driven from both the tendency of the decoherence rate to saturate at the lowest temperatures and also the absence of crossover from WL to WAL specific to conductors with strong spin-orbit scattering. Based on this observation, we conclude that spin memory of electrons in a field effect transistors based on epitaxial graphene with $n \sim 10^{11} \mathrm{~cm}^{-2}$ can exceed a micrometer scale, which proves the suitability of this system for spintronics applications.

The authors thank Floriana Lombardi, Thilo Bauch, and Per Delsing for helpful discussions. This work is supported by the Swedish Research Council and Foundation for Strategic Research, UK National Measurement Office and EU FP7 STREP ConceptGraphene.

*samuel.lara@chalmers.se

[1] Y.-M. Lin, C. Dimitrakopoulos, K. A. Jenkins, D. B. Farmer, H.-Y. Chiu, A. Grill, and P. Avouris, Science 327, 662 (2010).

[2] A. Tzalenchuk, S. Lara-Avila, A. Kalaboukhov, S. Paolillo, M. Syvajarvi, R. Yakimova, O. Kazakova, T. Janssen, V. Fal'ko, and S. Kubatkin, Nature Nanotech. 5, 186 (2010).

[3] S. Lara-Avila, K. Moth-Poulsen, R. Yakimova, T. Bjørnholm, V. Fal'ko, A. Tzalenchuk, and S. Kubatkin, Adv. Mater. 23, 878 (2011).

[4] T. J. B. M. Janssen, A. Tzalenchuk, R. Yakimova, S. Kubatkin, S. Lara-Avila, S. Kopylov, and V. I. Fal'ko, Phys. Rev. B 83, 233402 (2011).

[5] T. J. B. M. Janssen, N. E. Fletcher, R. Goebel, J. M. Williams, A. Tzalenchuk, R. Yakimova, S. Kubatkin, S. Lara-Avila, and V.I. Falko, New J. Phys. 13, 093026 (2011).

[6] F. V. Tikhonenko, D. W. Horsell, R. V. Gorbachev, and A. K. Savchenko, Phys. Rev. Lett. 100, 056802 (2008).

[7] F. V. Tikhonenko, A. A. Kozikov, A. K. Savchenko, and R. V. Gorbachev, Phys. Rev. Lett. 103, 226801 (2009).

[8] E. McCann, K. Kechedzhi, V. Falko, H. Suzuura, T. Ando, and B. Altshuler, Phys. Rev. Lett. 97, 14 (2006).

[9] V. Falko, K. Kechedzhi, E. McCann, B. Altshuler, H. Suzuura, and T. Ando, Solid State Commun. 143, 33 (2007).

[10] B. L. Altshuler, D. Khmelnitzkii, A. I. Larkin, and P. A. Lee, Phys. Rev. B 22, 5142 (1980).

[11] B. Altshuler and A. Aronov, in Electron-Electron InterActions in Disordered Systems, edited by A. Elfros and M. Pollak (North-Holland, Amsterdam, 1985).

[12] K. Nomura and A. H. MacDonald, Phys. Rev. Lett. 96, 256602 (2006).

[13] V. V. Cheianov and V. I. Falko, Phys. Rev. Lett. 97, 226801 (2006).

[14] E. H. Hwang and S. Das Sarma, Phys. Rev. B 75, 205418 (2007).

[15] S. Kopylov, A. Tzalenchuk, S. Kubatkin, and V. Fal'ko, Appl. Phys. Lett. 97, 112109 (2010).

[16] G. Bergmann, Phys. Rev. Lett. 48, 1046 (1982). 
[17] X. Wu, X. Li, Z. Song, C. Berger, and W. A. de Heer, Phys. Rev. Lett. 98, 136801 (2007).

[18] B. Jouault, B. Jabakhanji, N. Camara, W. Desrat, C. Consejo, and J. Camassel, Phys. Rev. B 83, 195417 (2011).

[19] T. Bauch, F. Lombardi, F. Tafuri, A. Barone, G. Rotoli, P. Delsing, and T. Claeson, Phys. Rev. Lett. 94, 087003 (2005).
[20] D.-K. Ki, D. Jeong, J.-H. Choi, H.-J. Lee, and K.-S. Park, Phys. Rev. B 78, 125409 (2008).

[21] E. H. Hwang and S. Das Sarma, Phys. Rev. B 77, 115449 (2008).

[22] H. Fukuyama, J. Phys. Soc. Jpn. 49, 644 (1980).

[23] A. A. Kozikov, A. K. Savchenko, B. N. Narozhny, and A. V. Shytov, Phys. Rev. B 82, 075424 (2010). 